\title{
On a derivation of the Goldstein-Einhorn probability weighting functions
}

\author{
JACEK CHUDZIAK(D
}

Dedicated to Professor Janos Aczél on the occasion of his 95-th birthday

\begin{abstract}
The well known Goldstein-Einhorn probability weighting functions have been characterized by Gonzalez and Wu using the linear in log odds preference condition. We provide an alternative way of deriving this class of functions. It is based on the concept of indifference prices.
\end{abstract}

Mathematics Subject Classification. 39B22, 91B16.

Keywords. Utility representation, Probability weighting function, Goldstein-Einhorn functions, Indifference pricing, Positive homogeneity.

\section{Introduction}

Many behavioral models of decision making under risk (e.g. rank-dependent utility, cumulative prospect theory) lead to the well known subjective utility representations involving a utility (or a value) function and a probability weighting function. The latter one is also called a probability distortion function. Possible forms of probability weighting functions have been investigated by several authors. Such functions are usually assumed to be non-decreasing and to satisfy the boundary conditions $w(0)=0$ and $w(1)=1$. The following classes of probability weighting functions are frequently used in a literature (cf. e.g. [3] and [11]):

- the Prelec function ([14])

$$
w(p)=\exp \left(-\alpha(-\ln p)^{\gamma}\right) \quad \text { for } \quad p \in(0,1], \quad \alpha, \gamma>0 ;
$$


- the $\mathrm{Wu}-$ Gonzalez function ([19])

$$
w(p)=\frac{p^{\gamma}}{\left(p^{\gamma}+(1-p)^{\gamma}\right)^{\eta}} \quad \text { for } \quad p \in[0,1], \quad \eta, \gamma>0
$$

- the Tversky-Kahneman function ([18])

$$
w(p)=\frac{p^{\gamma}}{\left(p^{\gamma}+(1-p)^{\gamma}\right)^{1 / \gamma}} \quad \text { for } \quad p \in[0,1], \quad \gamma>0 ;
$$

- the Goldstein-Einhorn function ([5])

$$
w(p)=\frac{\alpha p^{\gamma}}{\alpha p^{\gamma}+(1-p)^{\gamma}} \quad \text { for } \quad p \in[0,1], \quad \alpha, \gamma>0 .
$$

It is worth noting that Prelec [14] was the first to offer an axiomatic approach for estimating probability weighting functions. He derived the functions of the form (1) from a condition called compound invariance. Simpler axiomatizations than used in Prelec [14] was provided by Luce [11] and alNowaihi and Dhami [12]. Some problems concerning characterization of Prelec functions were investigated in Aczél and Luce [1]. It is remarkable that in Luce [11] some further methods for deriving probability weighting functions, namely $\phi$-reduction invariance and $\phi$-compound invariance, were suggested. It was shown in Luce [11] that, applying them, one can characterize several classes of probability weighting functions.

Probability weighting functions of the form (2) were introduced by Goldstein and Einhorn [5]. Some of their variants were applied e.g. by Birnbaum and McIntosh [2], Lattimore, Baker and Witte [10], Tversky and Fox [17]. The special case $\alpha=1$ has been used by Karmarkar [7,8]. Gonzalez and $\mathrm{Wu}[6]$ established a preference condition, called the linear-in-log-odds preference condition, that in the context of rank-dependent utility theory is necessary and sufficient for (2).

The aim of this paper is to give an alternative way of deriving probability weighting functions of the Goldstein-Einhorn type. It is based on the concept of indifference prices. Let us point out that the idea of using utility functions to price random outcomes has a long history. In modern economics literature utility based pricing dates back at least to Pratt [13]. For more details concerning utility based pricing we refer e.g. to Föllmer and Schied [4] and Raiffa [16].

We prove that, under the rank-dependent utility model, the positive homogeneity of the indifference bid price on the family of all binary lotteries characterizes not only the value functions but also the probability weighting functions. Furthermore, we show that this, rather surprising, property of bid prices can be applied to characterization of the Goldstein-Einhorn weighting function. The extension to three or more outcome gambles is investigated as well. 


\section{Indifference bid prices under rank-dependent utility}

Assume that $\mathcal{X}^{(f i n)}$ is a family of all gambles, that is finitely supported probability distributions on $\mathbb{R}$. For every $n \in \mathbb{N}$, with $n \geq 2$, let $\left\langle x_{1}, \ldots, x_{n} ; p_{1}, \ldots, p_{n}\right\rangle$ be a gamble in which consequences $x_{1}<\cdots<x_{n}$ occur with probabilities $p_{1}, \ldots, p_{n} \in(0,1)$, respectively. Denote by $\mathcal{X}^{(n)}$ a family of all such gambles. Furthermore, for every $x \in \mathbb{R}$, let $\langle x ; 1\rangle$ be a gamble offering a single payoff $x$ for sure. In the sequel, for $x \in \mathbb{R}$, we identify $\langle x ; 1\rangle$ with $x$. Assume that $\preceq$ is a preference relation on the family $\mathcal{X}^{(f i n)}$ and $\sim$ is the indifference relation on $\mathcal{X}^{(f i n)}$, induced by $\preceq$, that is, for every $X, Y \in \mathcal{X}^{(f i n)}$,

$$
X \sim Y \text { if and only if } X \preceq Y \text { and } Y \preceq X \text {. }
$$

One of the frequently used notions related to utility based pricing is the indifference bid price. In order to recall it, assume that $x_{0}$ is a decision maker's initial wealth. The indifference bid price for a given $X \in \mathcal{X}^{(f i n)}$ is a real number $P_{x_{0}}(X)$ such that

$$
x_{0}-P_{x_{0}}(X)+X \sim x_{0} .
$$

Condition (3) has the following interpretation. Suppose that an individual has to decide between entering into the gamble $X$ and rejecting it. The left hand side of (3) represents the case where the decision maker decides to play the gamble. In such a case the amount $P_{x_{0}}(X)$ is subtracted from the decision maker's initial wealth, who now has an opportunity to get one of the outcomes of the gamble. The right hand side of (3) represents the status quo. The decision maker rejects the gamble and remains with the initial wealth $x_{0}$. In general, the bid price need not exist and, even if it exists, it need not be unique. However, if $P_{x_{0}}(X)$ is uniquely determined, it expresses the maximal price at which the investor, with an initial wealth level $x_{0}$, is willing to buy $X$.

In what follows we analyze the bid price under rank-dependent utility, one of the most frequently applied behavioral alternatives of the classical expected utility model. Assume that the decision maker has a continuous strictly increasing value function $u: \mathbb{R} \rightarrow \mathbb{R}$, with $u(0)=0$, and a continuous non-decreasing weighting function $w:[0,1] \rightarrow[0,1]$, with $w(0)=0$ and $w(1)=1$. Then, for every $n \in \mathbb{N}$, a gamble $X=\left\langle x_{1}, \ldots, x_{n} ; p_{1}, \ldots, p_{n}\right\rangle \in \mathcal{X}^{(n)}$ is evaluated by the decision maker as (we adopt a convention $\sum_{i=n+1}^{n} p_{i}=0$ )

$$
\operatorname{RDU}(X)=\sum_{k=1}^{n}\left[w\left(\sum_{i=k}^{n} p_{i}\right)-w\left(\sum_{i=k+1}^{n} p_{i}\right)\right] u\left(x_{k}\right) .
$$

Therefore, for every $n \in \mathbb{N}$ and $X=\left\langle x_{1}, \ldots, x_{n} ; p_{1}, \ldots, p_{n}\right\rangle \in \mathcal{X}^{(n)}$, condition (3) is equivalent to

$$
\sum_{k=1}^{n}\left[w\left(\sum_{i=k}^{n} p_{i}\right)-w\left(\sum_{i=k+1}^{n} p_{i}\right)\right] u\left(x_{0}-P_{x_{0}}(X)+x_{k}\right)=u\left(x_{0}\right) .
$$


In the particular case $n=2$, for every $X=\left\langle x_{1}, x_{2} ; 1-p, p\right\rangle \in \chi^{(2)}$, we have

$$
(1-w(p)) u\left(x_{0}-P_{x_{0}}(X)+x_{1}\right)+w(p) u\left(x_{0}-P_{x_{0}}(X)+x_{2}\right)=u\left(x_{0}\right) .
$$

Since $u$ is continuous and strictly increasing, for every $n \in \mathbb{N}$ and $X=$ $\left\langle x_{1}, \ldots, x_{n} ; p_{1}, \ldots, p_{n}\right\rangle \in \mathcal{X}^{(n)}$ there exists a unique real number $P_{x_{0}}(X)$ such that (4) is valid. Hence, condition (4) defines in an implicit way a functional $P_{x_{0}}: \mathcal{X}^{(f i n)} \rightarrow \mathbb{R}$, which typically does not admit an explicit formula. Nevertheless, it follows from (4) that $P_{x_{0}}$ is a mean, i.e. for every $n \in \mathbb{N}$ and $X=\left\langle x_{1}, \ldots, x_{n} ; p_{1}, \ldots, p_{n}\right\rangle \in \mathcal{X}^{(n)}$ it holds that

$$
x_{1} \leq P_{x_{0}}\left(\left\langle x_{1}, \ldots, x_{n} ; p_{1}, \ldots, p_{n}\right\rangle\right) \leq x_{n} .
$$

Furthermore, $P_{x_{0}}$ is translation invariant, that is

$$
P_{x_{0}}\left(\left\langle x_{1}+z, \ldots, x_{n}+z ; p_{1}, \ldots, p_{n}\right\rangle\right)=P_{x_{0}}\left(\left\langle x_{1}, \ldots, x_{n} ; p_{1}, \ldots, p_{n}\right\rangle\right)+z
$$

for every $n \in \mathbb{N}, X=\left\langle x_{1}, \ldots, x_{n} ; p_{1}, \ldots, p_{n}\right\rangle \in \mathcal{X}^{(n)}$ and $z \in \mathbb{R}$.

Another desirable property of $P_{x_{0}}$ is positive homogeneity which, however, generally does not hold. Recall that $P_{x_{0}}$ is said to be positively homogeneous provided, for every $n \in \mathbb{N}, X=\left\langle x_{1}, \ldots, x_{n} ; p_{1}, \ldots, p_{n}\right\rangle \in \mathcal{X}^{(n)}$ and $\lambda \in[0, \infty)$, we have

$$
P_{x_{0}}\left(\left\langle\lambda x_{1}, \ldots, \lambda x_{n} ; p_{1}, \ldots, p_{n}\right\rangle\right)=\lambda P_{x_{0}}\left(\left\langle x_{1}, \ldots, x_{n} ; p_{1}, \ldots, p_{n}\right\rangle\right) .
$$

Positive homogeneity of $P_{x_{0}}$ implies that the pricing is currency-free, that is the indifference bid price does not depend on a currency in which the outcomes are expressed.

Remark 2.1. Assume that $P_{x_{0}}$ is positively homogeneous on $\mathcal{X}^{(2)}$, that is, for every $\left\langle x_{1}, x_{2} ; 1-p, p\right\rangle \in \mathcal{X}^{(2)}$ and $\lambda \in(0, \infty)$, it holds that

$$
P_{x_{0}}\left(\left\langle\lambda x_{1}, \lambda x_{2} ; 1-p, p\right\rangle\right)=\lambda P_{x_{0}}\left(\left\langle x_{1}, x_{2} ; 1-p, p\right\rangle\right) .
$$

Then, taking $x_{1}=0$ and $x_{2}=1$, we obtain

$$
P_{x_{0}}(\langle 0, \lambda ; 1-p, p\rangle)=\lambda P_{x_{0}}(\langle 0,1 ; 1-p, p\rangle) \text { for } \lambda \in(0, \infty), p \in(0,1) .
$$

Moreover, in view of $(6)$, we have $P_{x_{0}}(\langle 0,1 ; 1-p, p\rangle) \in[0,1]$ for $p \in(0,1)$. Hence

$$
P_{x_{0}}(\langle 0, x ; 1-p, p\rangle)=f(p) x \quad \text { for } \quad x \in(0, \infty), p \in(0,1),
$$

where $f:(0,1) \rightarrow[0,1]$ is given by

$$
f(p)=P_{x_{0}}(\langle 0,1 ; 1-p, p\rangle) \text { for } p \in(0,1) .
$$

Furthermore, taking (7) into account, for every $\left\langle x_{1}, x_{2} ; 1-p, p\right\rangle \in \mathcal{X}^{(2)}$, we get

$P_{x_{0}}\left(\left\langle x_{1}, x_{2} ; 1-p, p\right\rangle\right)=P_{x_{0}}\left(\left\langle 0, x_{2}-x_{1} ; 1-p, p\right\rangle\right)+x_{1}=f(p)\left(x_{2}-x_{1}\right)+x_{1}$. 
Thus

$P_{x_{0}}\left(\left\langle x_{1}, x_{2} ; 1-p, p\right\rangle\right)=(1-f(p)) x_{1}+f(p) x_{2} \quad$ for $\quad\left\langle x_{1}, x_{2} ; 1-p, p\right\rangle \in \mathcal{X}^{(2)}$.

\section{Results}

In the whole section we assume that a decision maker, having an initial wealth $x_{0}$, a continuous strictly increasing value function $u: \mathbb{R} \rightarrow \mathbb{R}$, with $u(0)=0$, and a continuous non-decreasing weighting function $w:[0,1] \rightarrow[0,1]$ such that $w(0)=0$ and $w(1)=1$, follows rank-dependent utility.

The first result characterizes those indifference bid prices whose restriction to the family $\mathcal{X}^{(2)}$ is of the form (9). As we have already mentioned, in the Introduction, this characterization involves not only the value function but also the probability weighting function.

Theorem 3.1. Let $f:(0,1) \longrightarrow[0,1]$. The following two statements are equivalent:

(i) $P_{x_{0}}$ is of the form (9);

(ii) there exist $\alpha, \beta, \gamma \in(0, \infty)$ and $\delta \in \mathbb{R}$ such that

$$
u(x)= \begin{cases}-\alpha \beta\left(x_{0}-x\right)^{\gamma}+\delta \text { for } x \in\left(-\infty, x_{0}\right], \\ \beta\left(x-x_{0}\right)^{\gamma}+\delta & \text { for } x \in\left(x_{0}, \infty\right)\end{cases}
$$

and

$$
w(p)=\frac{\alpha f(p)^{\gamma}}{\alpha f(p)^{\gamma}+(1-f(p))^{\gamma}} \quad \text { for } \quad p \in(0,1) .
$$

Proof. Assume that $(i)$ holds. Then, applying (5) and (9), for every $\left\langle x_{1}, x_{2} ; 1-\right.$ $p, p\rangle \in \mathcal{X}^{(2)}$, we obtain

$$
(1-w(p)) u\left(x_{0}+f(p)\left(x_{1}-x_{2}\right)\right)+w(p) u\left(x_{0}+(1-f(p))\left(x_{2}-x_{1}\right)\right)=u\left(x_{0}\right) .
$$

Hence, taking $x_{2}=x_{1}+x$, where $x \in(0, \infty)$, we conclude that

$$
(1-w(p)) v(-f(p) x)+w(p) v((1-f(p)) x)=0 \text { for } x \in(0, \infty), p \in(0,1)
$$

where $v: \mathbb{R} \rightarrow \mathbb{R}$ is given by

$$
v(x):=u\left(x_{0}+x\right)-u\left(x_{0}\right) \text { for } x \in \mathbb{R} .
$$

Obviously $v(0)=0$ and, as $u$ is continuous and strictly increasing, so is $v$. Therefore, since $w$ is continuous, with $w(0)=0$ and $w(1)=1$, from (12) we derive that

$$
\lim _{p \rightarrow p_{w}^{+}} f(p)=0, \quad \text { and } \quad \lim _{p \rightarrow 1^{-}} f(p)=1
$$


where $p_{w}:=\inf \{p \in(0,1]: w(p)>0\}$. Furthermore, if $f\left(p_{0}\right)=0$ for some $p_{0} \in(0,1)$ then, in view of $(12)$, we get $w\left(p_{0}\right) v(x)=0$ for $x \in \mathbb{R}$. Thus $w\left(p_{0}\right)=0$, that is $p_{0} \leq p_{w}$. In this way we have proved that

$$
f(p)>0 \text { for } p \in\left(p_{w}, 1\right] .
$$

So, replacing $x$ by $x / f(p)$ in (12), we get

$$
v\left(\frac{1-f(p)}{f(p)} x\right)=-\frac{1-w(p)}{w(p)} v(-x) \text { for } x \in(0, \infty), p \in\left(p_{w}, 1\right) .
$$

Applying (16) with $x=1$ and using the fact that $v(-1) \neq 0$, we obtain

$$
\frac{1-w(p)}{w(p)}=-\frac{1}{v(-1)} v\left(\frac{1-f(p)}{f(p)}\right) \text { for } p \in\left(p_{w}, 1\right) .
$$

Hence, (16) becomes

$$
v\left(\frac{1-f(p)}{f(p)} x\right)=\frac{1}{v(-1)} v\left(\frac{1-f(p)}{f(p)}\right) v(-x) \text { for } x \in(0, \infty), p \in\left(p_{w}, 1\right) .
$$

Since $v$ and $w$ are continuous and $v$ is strictly increasing, it follows from (16) that $f_{\mid\left(p_{w}, 1\right)}$ is continuous. Thus, taking (14)-(15) into account, we get

$$
(0, \infty) \subseteq\left\{\frac{1-f(p)}{f(p)}: p \in\left(p_{w}, 1\right)\right\} .
$$

Therefore, as $v(0)=0$, making use of (17), we obtain

$$
\phi(x y)=\psi(x) \phi(y) \text { for } x, y \in[0, \infty),
$$

where $\phi, \psi:[0, \infty) \rightarrow \mathbb{R}$ are given by

$$
\phi(x):=\frac{1}{v(-1)} v(x) \text { for } x \in[0, \infty)
$$

and

$$
\psi(x):=\frac{1}{v(-1)} v(-x) \text { for } x \in[0, \infty) .
$$

Note that $\phi$ and $\psi$ are continuous and, as $v(-1)<0, \phi$ is strictly decreasing, while $\psi$ is strictly increasing. Thus, applying Theorem 13.3.8 in Kuczma [9], we conclude that there exist $a \in(-\infty, 0)$ and $\gamma \in(0, \infty)$ such that

$$
\phi(x)=a x^{\gamma} \text { for } x \in[0, \infty)
$$

and

$$
\psi(x)=x^{\gamma} \text { for } \quad x \in[0, \infty) .
$$

Hence, from (18)-(19), we derive that

$$
v(x)= \begin{cases}v(-1)(-x)^{\gamma} & \text { for } x \in(-\infty, 0], \\ a v(-1) x^{\gamma} & \text { for } x \in(0, \infty) .\end{cases}
$$


Moreover, inserting $v$ of the form (20) into (12), for every $x \in(0, \infty)$ and $p \in(0,1)$, we get

$$
v(-1)(1-w(p))(f(p) x)^{\gamma}+a v(-1) w(p)((1-f(p)) x)^{\gamma}=0 .
$$

Solving this equation for $w(p)$ gives

$$
w(p)=\frac{-(1 / a) f(p)^{\gamma}}{-(1 / a) f(p)^{\gamma}+(1-f(p))^{\gamma}} \quad \text { for } \quad p \in(0,1) .
$$

Therefore, taking (13) and (20) into account, we obtain (10)-(11) with $\alpha:=$ $-1 / a, \beta:=a v(-1)$ and $\delta:=u\left(x_{0}\right)$. Note also that as $a \in(-\infty, 0)$ and $v(-1)<$ 0 , we have $\alpha, \beta \in(0, \infty)$. Thus, the implication (i) $\Longrightarrow$ (ii) is proved.

If (ii) holds then, inserting into (5) $u$ and $w$ of the form (10) and (11), respectively, making use of (6) and solving equation obtained in this way with respect to $P_{x_{0}}(X)$, we get $(9)$. This proves the implication (ii) $\Longrightarrow(\mathrm{i})$.

The following result, showing an alternative way to derive the GoldsteinEinhorn probability weighting function under rank-dependent utility, is a direct consequence of Theorem 3.1.

Theorem 3.2. The following statements are equivalent:

(i) the indifference bid price for every binary gamble coincides with the expected value of the gamble;

(ii) there exist $\alpha, \beta, \gamma \in(0, \infty)$ and $\delta \in \mathbb{R}$ such that $u$ is of the form (10) and

$$
w(p)=\frac{\alpha p^{\gamma}}{\alpha p^{\gamma}+(1-p)^{\gamma}} \text { for } p \in[0,1] .
$$

It is a natural question, whether the result presented above has an extension to three or more outcome gambles. The following theorem answers the question.

Theorem 3.3. Let $n \in \mathbb{N}$ be greater than 2. Then, under rank-dependent utility, the indifference bid price for every gamble in $\mathcal{X}^{(n)}$ coincides with the expected value of the gamble if and only if the weighting function is the identity on $[0,1]$ and the value function is linear.

Proof. If the weighting function is the identity on $[0,1]$ and the value function is linear then, in view of (4), we get

$$
P_{x_{0}}\left(\left\langle x_{1}, \ldots, x_{n} ; p_{1}, \ldots, p_{n}\right\rangle\right)=\sum_{k=1}^{n} p_{k} x_{k} \quad \text { for } \quad\left\langle x_{1}, \ldots, x_{n} ; p_{1}, \ldots, p_{n}\right\rangle \in \mathcal{X}^{(n)} \text {. }
$$

Thus, the indifference bid price for every gamble in $\mathcal{X}^{(n)}$ coincides with the expected value of the gamble. 
In order to prove a converse implication, assume that (23) holds. Then, for every $x, y, z \in(0, \infty)$ such that $(n-2) x<y<z$, we have

$$
P_{x_{0}}(\langle x, 2 x, \ldots,(n-2) x, y, z ; 1 / n, \ldots, 1 / n\rangle)=\frac{(n-1)(n-2)}{2 n} x+\frac{y+z}{n} .
$$

Hence, taking (4) into account, we obtain

$$
\begin{aligned}
& \sum_{k=1}^{n-2}\left[w\left(\frac{n+1-k}{n}\right)-w\left(\frac{n-k}{n}\right)\right] u\left(x_{0}+k x-\frac{(n-1)(n-2)}{2 n} x-\frac{y+z}{n}\right) \\
& \quad+\left[w\left(\frac{2}{n}\right)-w\left(\frac{1}{n}\right)\right] u\left(x_{0}+y-\frac{(n-1)(n-2)}{2 n} x-\frac{y+z}{n}\right) \\
& \quad+w\left(\frac{1}{n}\right) u\left(x_{0}+z-\frac{(n-1)(n-2)}{2 n} x-\frac{y+z}{n}\right)=u\left(x_{0}\right) .
\end{aligned}
$$

Thus, as $u$ is continuous, letting $x \rightarrow 0^{+}$, for every $y, z \in(0, \infty)$ with $y<z$, we get

$$
\alpha v\left(-\frac{y+z}{n}\right)+\beta v\left(\left(1-\frac{1}{n}\right) y-\frac{z}{n}\right)+(1-\alpha-\beta) v\left(\left(1-\frac{1}{n}\right) z-\frac{y}{n}\right)=0
$$

where $\alpha:=1-w(2 / n), \beta:=w(2 / n)-w(1 / n)$ and $v: \mathbb{R} \rightarrow \mathbb{R}$ is given by (13). Furthermore, taking $(a, b) \in D$, where

$$
D:=\left\{(a, b) \in \mathbb{R}^{2}:-b /(n-1)<a<b\right\}
$$

and setting

$$
y=\frac{(n-1) a+b}{n-2} \text { and } z=\frac{a+(n-1) b}{n-2},
$$

we obtain $0<y<z$,

$$
\left(1-\frac{1}{n}\right) y-\frac{z}{n}=a,\left(1-\frac{1}{n}\right) z-\frac{y}{n}=b \text { and }-\frac{y+z}{n}=\frac{a+b}{2-n} .
$$

Therefore, in view of (24), we get

$$
\beta v(a)+(1-\alpha-\beta) v(b)=-\alpha v((a+b) /(2-n)) \quad \text { for } \quad(a, b) \in D .
$$

Note that, by (13), $v$ is strictly increasing and continuous, with $v(0)=0$. Moreover, we have

$$
D_{1}:=\{a \in \mathbb{R}:(a, b) \in D \text { for some } b \in \mathbb{R}\}=\mathbb{R} .
$$

Thus, applying Theorem 1 in Radó and Baker [15] and Theorem 13.3.5 in Kuczma [9], we obtain that there exists $c \in \mathbb{R}$ such that

$$
\beta v(x)=c x \text { for } \quad x \in \mathbb{R} .
$$


Suppose that $\beta=0$. Then, as $((2-n) z-1,1) \in D$ for $z \in\left(\frac{2}{2-n}, \frac{1}{1-n}\right)$, setting $a=(2-n) z-1$ and $b=1$ in $(25)$, we get

$$
(1-\alpha) v(1)+\alpha v(z)=0 \text { for } z \in\left(\frac{2}{2-n}, \frac{1}{1-n}\right) .
$$

Since $v$ is strictly increasing, with $v(0)=0$, this yields a contradiction. Therefore $\beta \neq 0$ and so, by (26), $v$ is linear. Thus, as $u(0)=0$, from (13) we deduce that $u$ is linear. Hence, taking (4) into account, for every $\left\langle x_{1}, \ldots, x_{n} ; p_{1}, \ldots, p_{n}\right\rangle$ $\in \mathcal{X}^{(n)}$, we obtain

$$
P\left(\left\langle x_{1}, \ldots, x_{n} ; p_{1}, \ldots, p_{n}\right\rangle\right)=\sum_{k=1}^{n}\left[w\left(\sum_{i=k}^{n} p_{i}\right)-w\left(\sum_{i=k+1}^{n} p_{i}\right)\right] x_{k}
$$

which, together with (23), for every $\left\langle x_{1}, \ldots, x_{n} ; p_{1}, \ldots, p_{n}\right\rangle \in \chi^{(n)}$ gives

$$
\sum_{k=1}^{n}\left[w\left(\sum_{i=k}^{n} p_{i}\right)-w\left(\sum_{i=k+1}^{n} p_{i}\right)-p_{k}\right] x_{k}=0
$$

Let $p \in(0,1)$ and $q:=(1-p) /(n-1)$. Then, for every $x \in(0,1 /(n-1))$, we have

$$
\langle x, 2 x, \ldots,(n-1) x, 1 ; q, q, \ldots, q, p\rangle \in \mathcal{X}^{(n)}
$$

and so, in view of (27), we obtain

$$
\sum_{k=1}^{n-1}[w((n-k) q+p)-w((n-k-1) q+p)-q] k x+w(p)-p=0 .
$$

Passing in this equality to the limit with $x \rightarrow 0^{+}$, we get $w(p)=p$. In this way we have proved that $w(p)=p$ for $p \in(0,1)$. Since $w(0)=0$ and $w(1)=1$, this means that $w$ is the identity on $[0,1]$.

\section{Conclusion}

It is known that the Goldstein-Einhorn probability weighting function can be characterized by the so called linear in log-odds-preference condition. We propose an alternative way of deriving this class of weighting functions. It is based on the concept of indifference prices. We have proved that, under the rank-dependent utility model, a necessary and sufficient condition for the indifference bid price to coincide with the expected value for binary gambles is that the weighting function is of the Goldstein-Einhorn type and the utility function is power, with coefficients strictly related to those of the weighting function. Furthermore we have shown that this phenomenon disappears in the 
case of three or more outcome gambles. Namely, in such a setting the indifference bid price coincides with the expected value if and only if the weighting function is the identity on $[0,1]$ and the value function is linear.

Open Access. This article is licensed under a Creative Commons Attribution 4.0 International License, which permits use, sharing, adaptation, distribution and reproduction in any medium or format, as long as you give appropriate credit to the original author(s) and the source, provide a link to the Creative Commons licence, and indicate if changes were made. The images or other third party material in this article are included in the article's Creative Commons licence, unless indicated otherwise in a credit line to the material. If material is not included in the article's Creative Commons licence and your intended use is not permitted by statutory regulation or exceeds the permitted use, you will need to obtain permission directly from the copyright holder. To view a copy of this licence, visit http:// creativecommons.org/licenses/by/4.0/.

Publisher's Note Springer Nature remains neutral with regard to jurisdictional claims in published maps and institutional affiliations.

\section{References}

[1] Aczél, J., Luce, D.: A behavioral condition for Prelec's weighting function on the positive line without assuming $W(1)=1$. J. Math. Psychol. 51, 126-129 (2007)

[2] Birnbaum, M.H., McIntosh, W.R.: Violations of branch independence in choices between gamles. Organ. Behav. Hum. Decis. Processes 67, 91-110 (1996)

[3] Chechile, R.A., Barch, D.H.: Using logarithmic derivative functions for assesing the risky weighting functions for binary gambles. J. Math. Psychol. 57, 15-28 (2013)

[4] Föllmer, H., Schied, A.: Stochastic Finance. Walter de Gruyter \& Co., Berlin (2004)

[5] Goldstein, W.M., Einhorn, H.J.: Expression theory and the preference reversal phenomenon. Psychol. Rev. 94, 236-254 (1987)

[6] Gonzalez, R., Wu, G.: On the shape of the probability weighting function. Cognit. Psychol. 38, 129-166 (1999)

[7] Karmarkar, U.: Subjectively weighted utility: a descriptive extension of the expected utility model. Organ. Behav. Hum. Perform. 21, 61-72 (1978)

[8] Karmarkar, U.: Subjectively weighted utility and the Allais paradox. Organ. Behav. Hum. Perform. 24, 67-72 (1979)

[9] Kuczma, M.: An Introduction to the Theory of Functional Equations and Inequalities. Birkhäuser, Berlin (2009)

[10] Lattimore, P.K., Baker, J.R., Witte, A.D.: The influence of probability on risky choice: a parametric examination. J. Econ. Behav. Organ. 17, 377-400 (1992)

[11] Luce, D.: Reduction invariance and Prelec's weighting functions. J. Math. Psychol. 45, 167-179 (2001)

[12] al-Nowaihi, A., Dhami, S.: A simple derivation of Prelec's probability weighting function. J. Math. Psychol. 50, 521-524 (2006)

[13] Pratt, J.W.: Risk aversion in the small and in the large. Econometrica 32, 122-136 (1964)

[14] Prelec, D.: The probability weighting functions. Econometrica 66, 497-527 (1998)

[15] Radó, F., Baker, J.A.: Pexider's equation and aggregation of allocations. Aequ. Math. 32, 227-239 (1987)

[16] Raiffa, H.: Decision Analysis: Introductory Lectures on Choices Under Uncertainty. Addison-Wesley, Boston (1968) 
[17] Tversky, A., Fox, C.R.: Weighting risk and uncertainty. Psychol. Rev. 102, 269-283 (1995)

[18] Tversky, A., Kahneman, D.: Advances in prospect theory: cumulative representation of uncertainty. J. Risk Uncertain. 5, 297-323 (1992)

[19] Wu, G., Gonzalez, R.: Curvature of the probability weighting function. Manag. Sci. 42, 1676-1690 (1996)

Jacek Chudziak

Institute of Mathematics

University of Rzeszów

ul. Pigonia 1

35-310 Rzeszow

Poland

e-mail: chudziak@ur.edu.pl

Received: September 4, 2019

Revised: January 4, 2020 